Photo-Print (Vilber Lourmat IP-008-SD) دستخاه

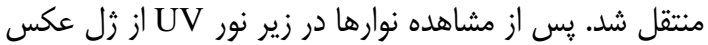

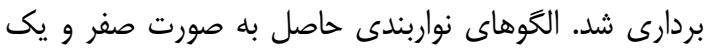

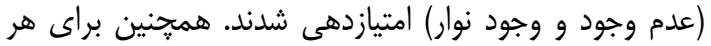

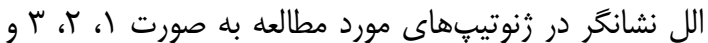

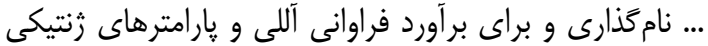

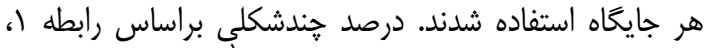

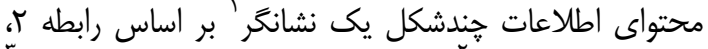

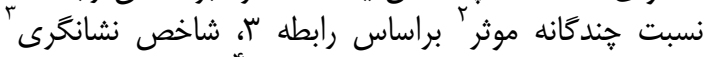

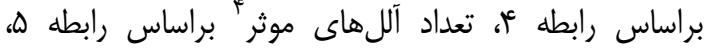

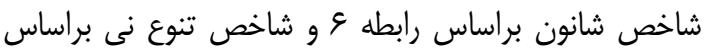

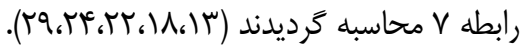

$\left(\frac{n p}{n}\right) * 100$

$1-\sum_{i=1}^{n} p_{i}$

$\mathrm{EMR}=\mathrm{n}_{\mathrm{p}} \times \beta$

PIC $\times$ EMR

$\frac{1}{\sum p_{i}^{2}}$

$-\sum_{i=1}^{n} p_{\bar{i}} \ln p_{\bar{i}}$

$-\ln \frac{J x y}{\sqrt{J x J y}}$

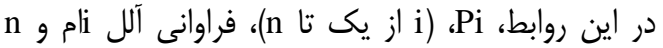

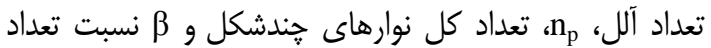

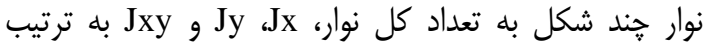

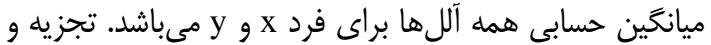

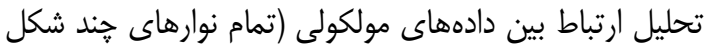

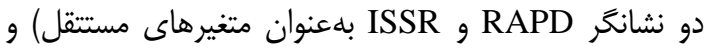

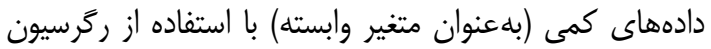

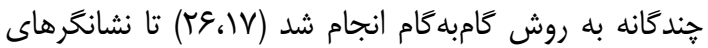

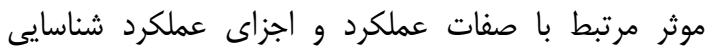

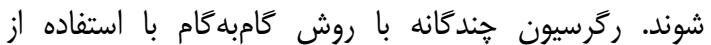

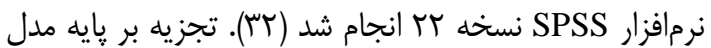

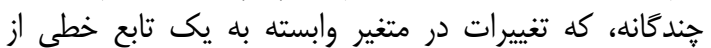
مجموعهاى از متغيرهاى مستقل (هاّ) به صورت رابط رابطه زير

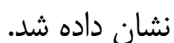

$\mathrm{Y}=\mathrm{a}+\mathrm{b}_{1} \mathrm{~m}_{1}+\cdots+\mathrm{b}_{\mathrm{j}} \mathrm{m}_{\mathrm{j}}+\cdots+\mathrm{b}_{\mathrm{n}} \mathrm{m}_{\mathrm{n}}+\mathrm{d}+\mathrm{e}$

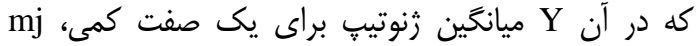

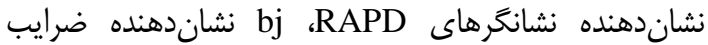

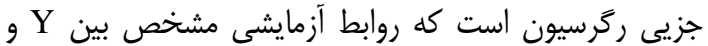

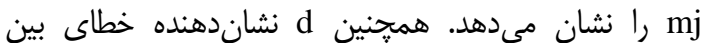

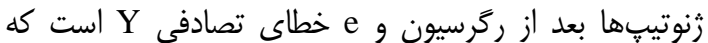

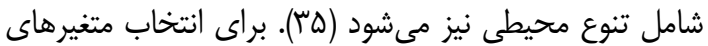

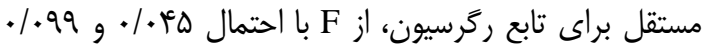

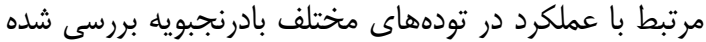

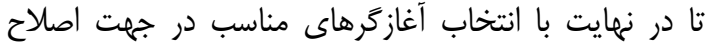
بادرنجبويه كامى موثر برداشت.

\section{مواد و روشها}

در اين تحقيق، تعداد باد إزنوتيب بادرنجبويه كه از بخش

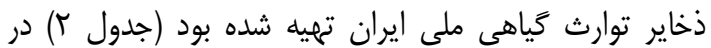

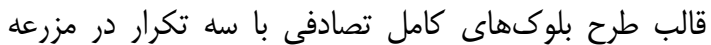

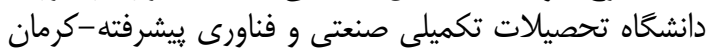

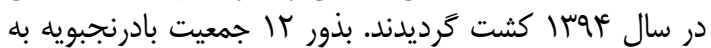

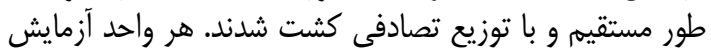

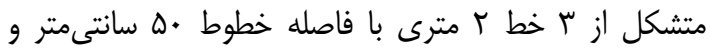

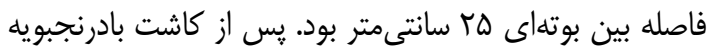

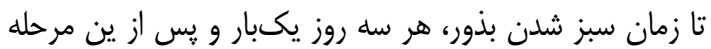

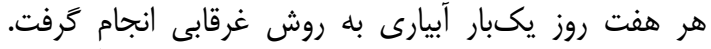

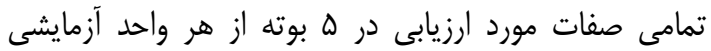

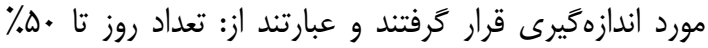

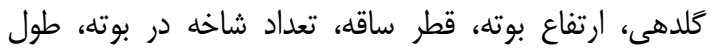

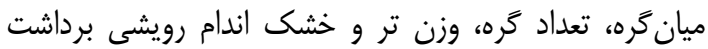

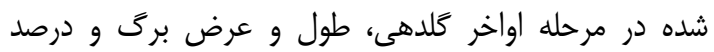

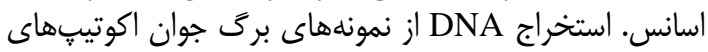

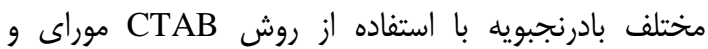

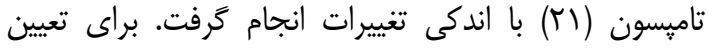

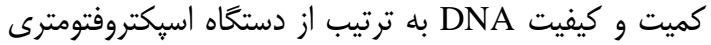

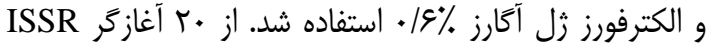

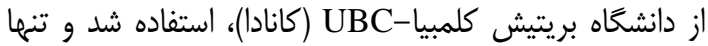

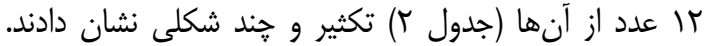

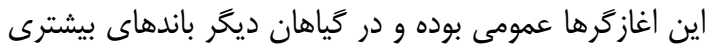

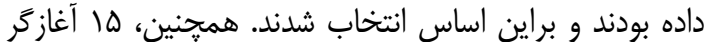

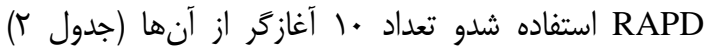

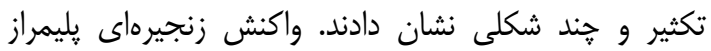

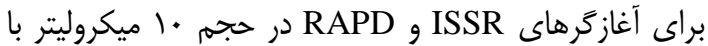

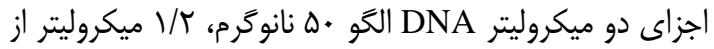

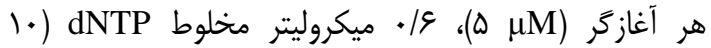

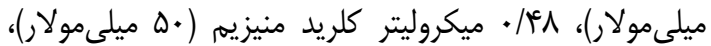

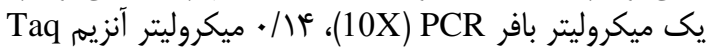

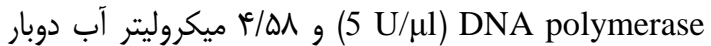
تقطير شده انجام شد. קرخه حرارتى شارل شامل ه دقيقه واسرشته

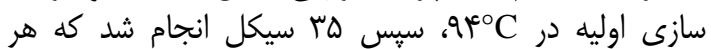

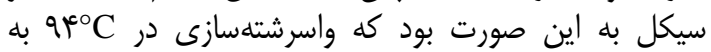

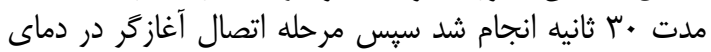

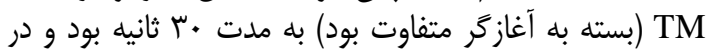

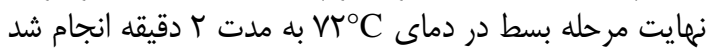

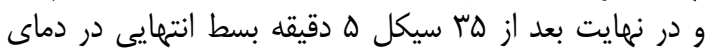

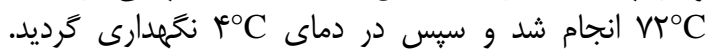

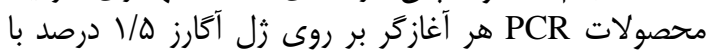

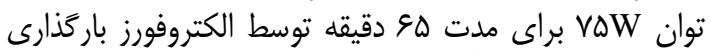

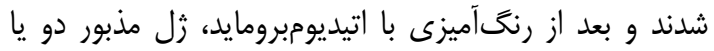
سه مرتبه با آب معمولى شستشو داده شد. سيس زل زل به 


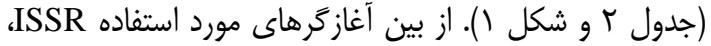

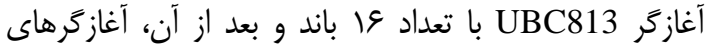

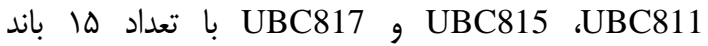

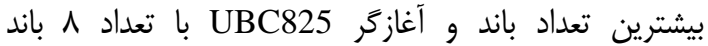

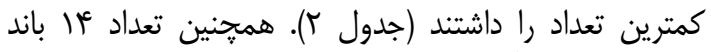

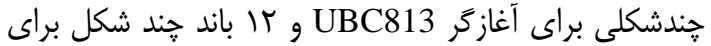

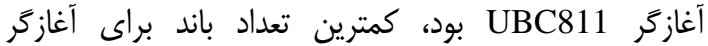

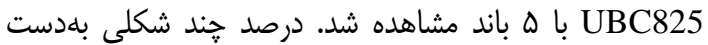

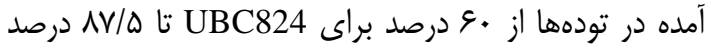
براى UBC813 متغيير بود.
به ترتيب براى وارد كردن و خارج كردن متغيرها استفاده كرديد.

نتايج و بحث

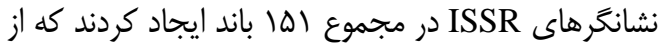

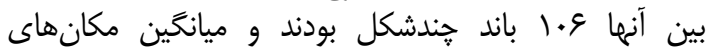

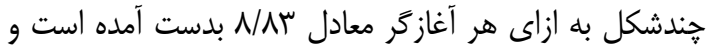

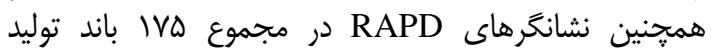

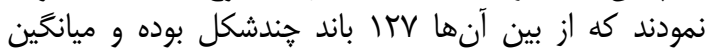

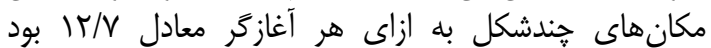

Table 1. Collecting place and code number of lemon balm ecotypes

\begin{tabular}{|c|c|c|c|}
\hline Code & Collection region & Code & Collection region \\
\hline G1 & $\begin{array}{c}\text { تهران- دماوند } \\
\text { Tehran-Damavand }\end{array}$ & G7 & $\begin{array}{c}\text { Kerman } \\
\text { Kerman }\end{array}$ \\
\hline $\mathrm{G} 2$ & $\begin{array}{c}\text { اردبيل } \\
\text { Ardabil }\end{array}$ & G8 & $\begin{array}{c}\text { اصفهان- يزدآباد } \\
\text { Isfahan -YazdAbad }\end{array}$ \\
\hline G3 & $\begin{array}{c}\text { اصفهان-نجفآباد -NajafAbad } \\
\text { Isfahan - }\end{array}$ & G9 & $\begin{array}{c}\text { خراسان جنوبى- سربيشه } \\
\text { South Khorasan-Sarbisheh }\end{array}$ \\
\hline G4 & $\begin{array}{l}\text { قزوين-يك } \\
\text { Qazvin-1 }\end{array}$ & G10 & $\begin{array}{c}\text { آذربايجان شرقىى } \\
\text { East Azerbaijan }\end{array}$ \\
\hline G5 & فارس & G11 & قزوين-دو \\
\hline G6 & $\begin{array}{c}\text { همدان- همدان } \\
\text { Hamedan-Hamedan }\end{array}$ & G12 & $\begin{array}{l}\text { كيلان-لاهيجان } \\
\text { Guilan-Lahijan }\end{array}$ \\
\hline
\end{tabular}

جدول r- مشخصات نشانگرهاى ISSR و RAPD شاخصهاى نشانخرى در تودههاى بادرنجبويه

Table 2. ISSR and RAPD primers characteristics and marker indices in lemon balm ecotypes

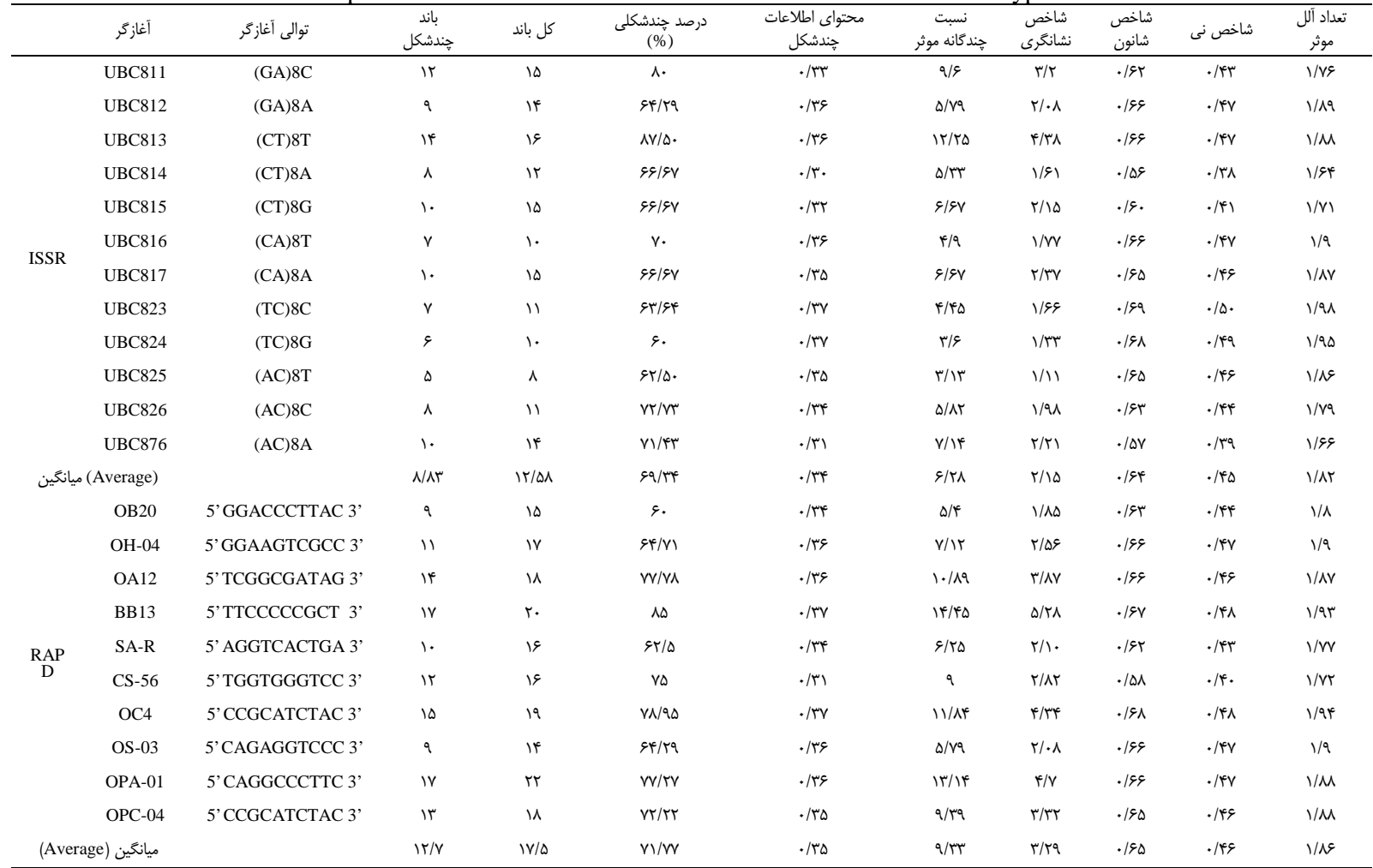




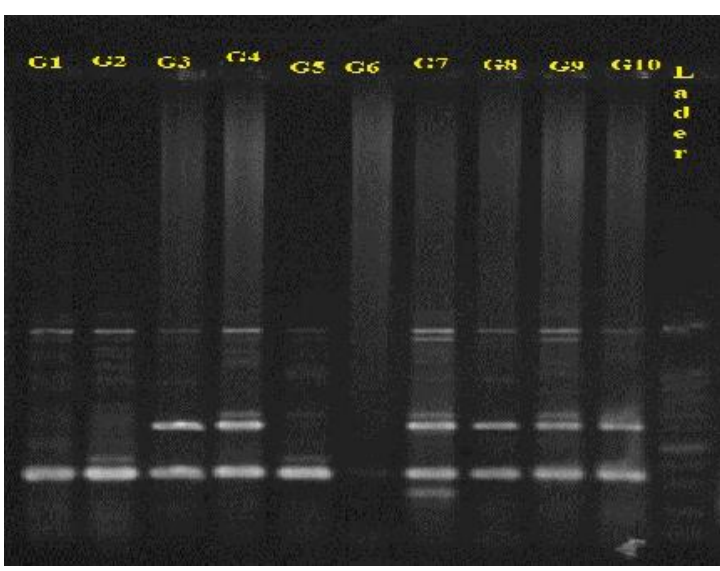

ب

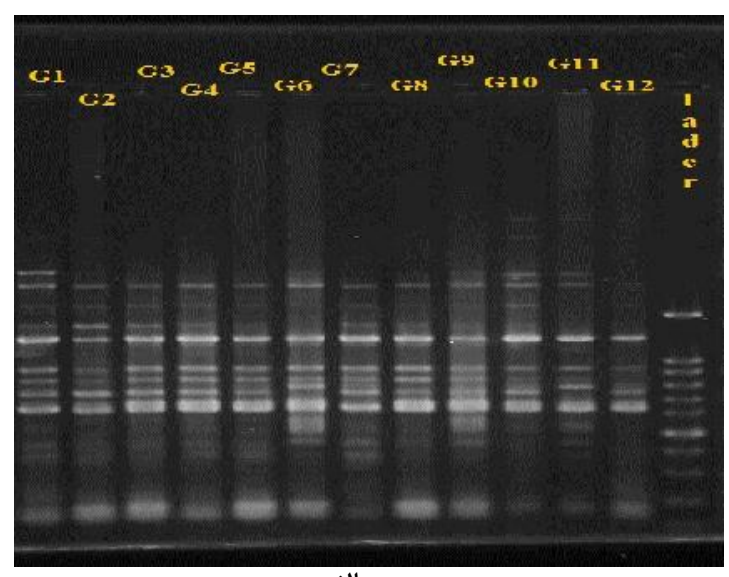

الف

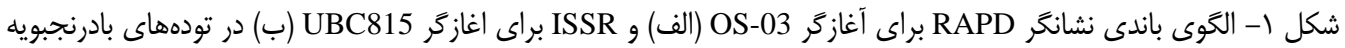

Figure 1. Primer banding pattern of RAPD for OS-03 primer (a) and ISSR for UBC815 primer (b) in lemon balm ecotypes.

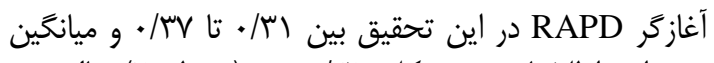

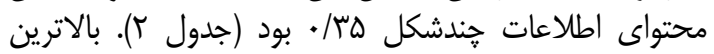
ميزان PIC براى آغازكر ISSR در آغازكرهاى

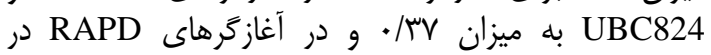

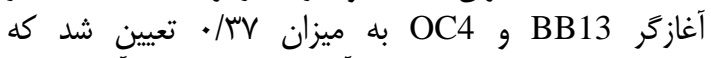

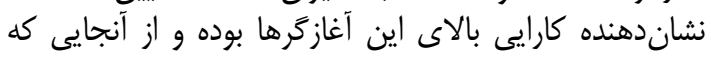

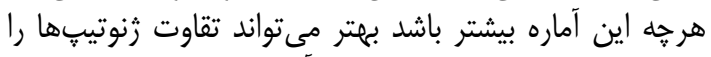

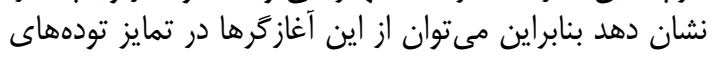

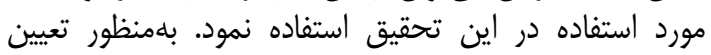

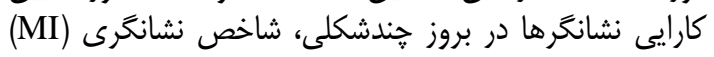

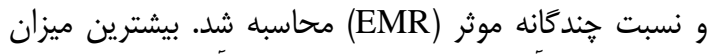

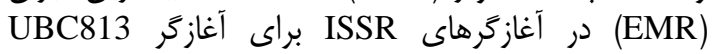

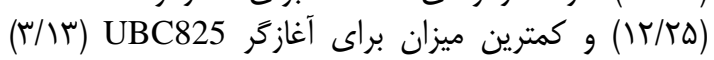

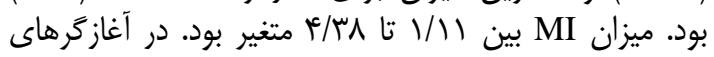

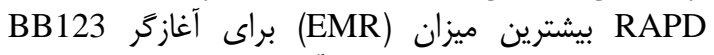

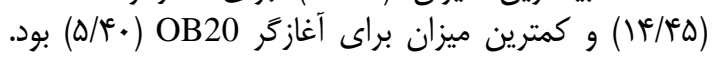

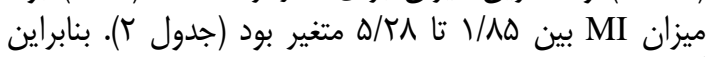

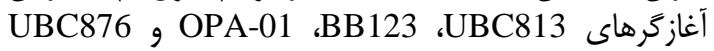
بيشترين كارايى را در بين نشانكرها

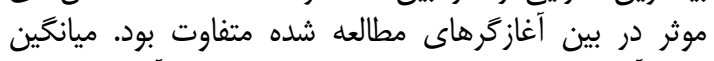

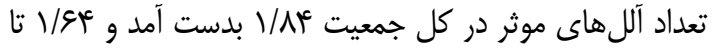

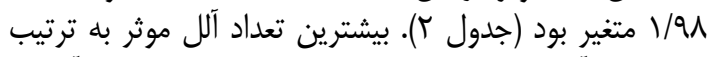

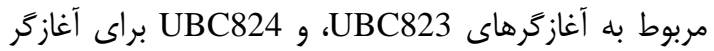

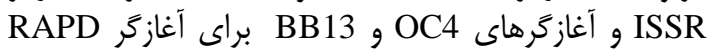

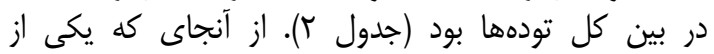

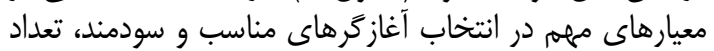

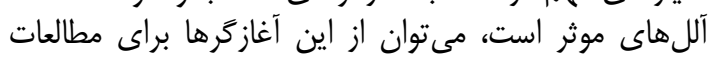

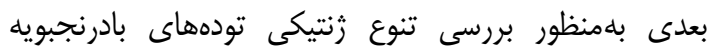

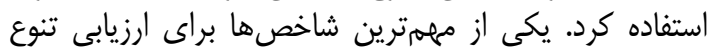

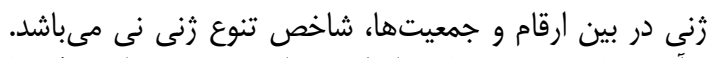

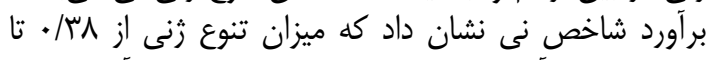

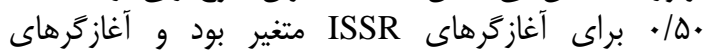

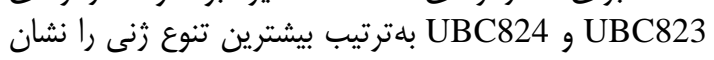

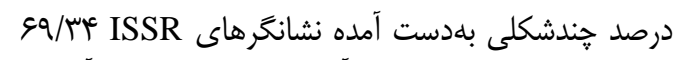

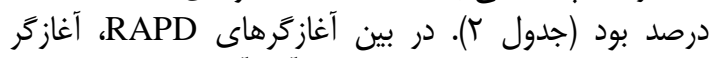

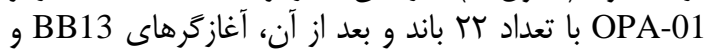

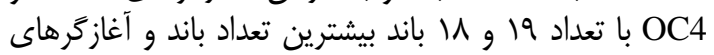

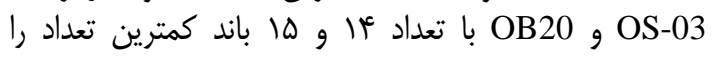

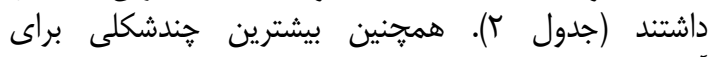

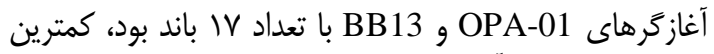

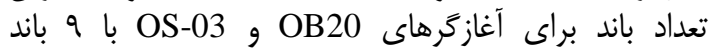

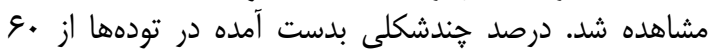

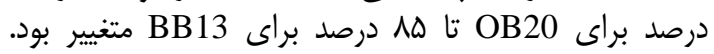

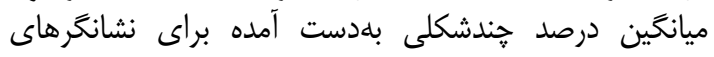

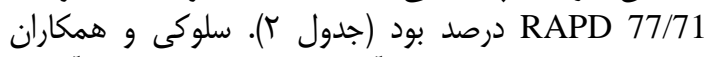

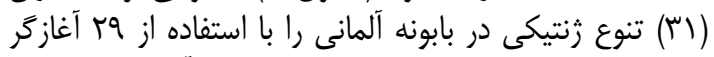

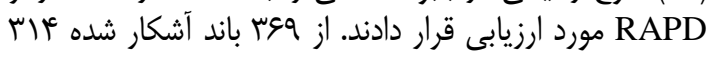

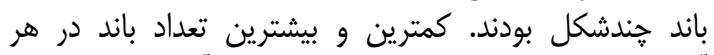

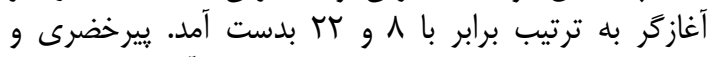

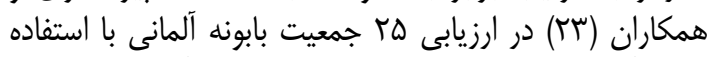

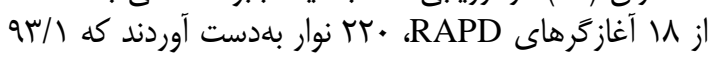

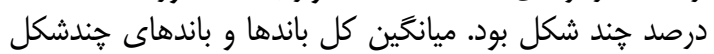

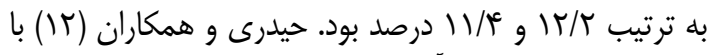
استفاده از جهار تركيب آغازگر

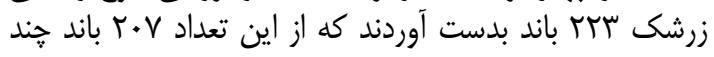

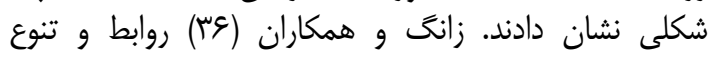

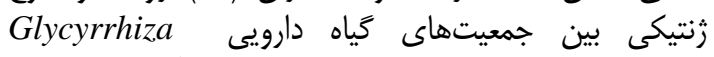

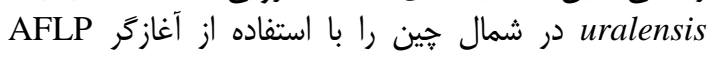

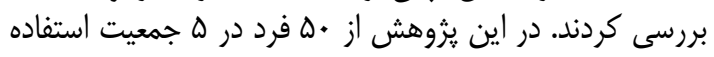

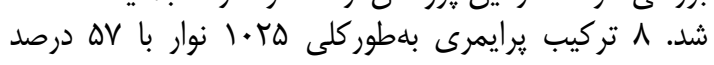

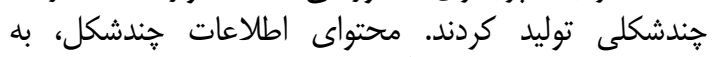

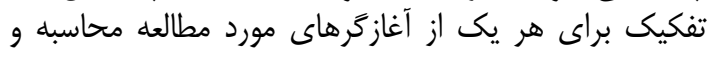

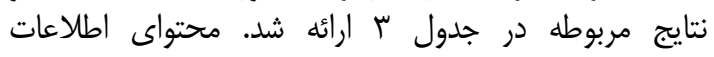

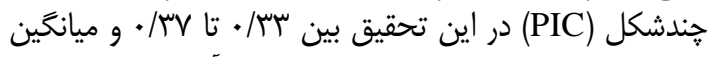

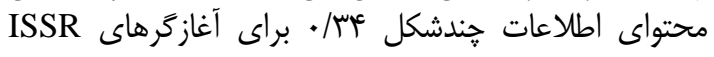

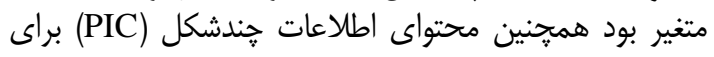




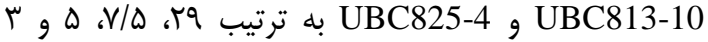

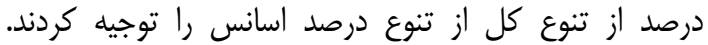

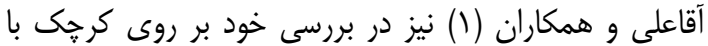

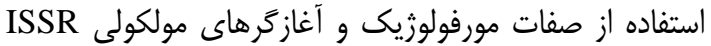

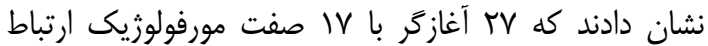

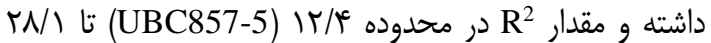

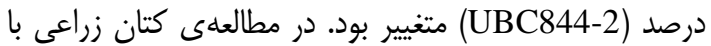

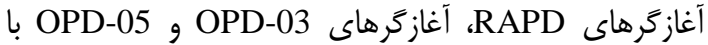

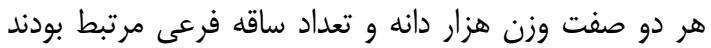

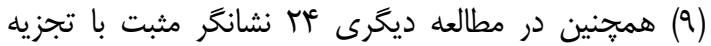

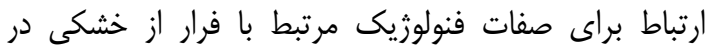

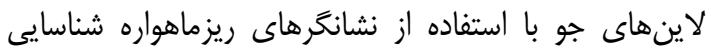

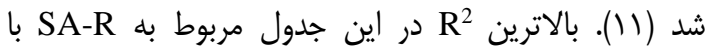

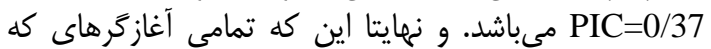

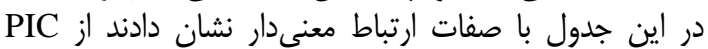

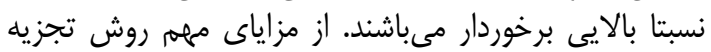

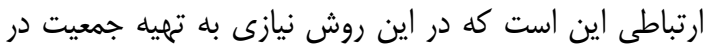

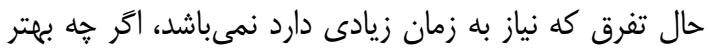

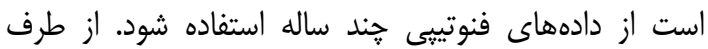

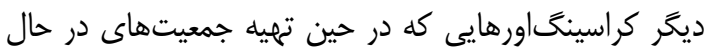

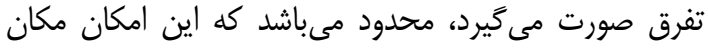

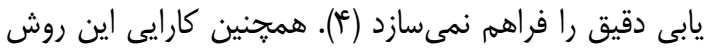

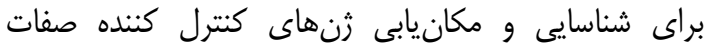

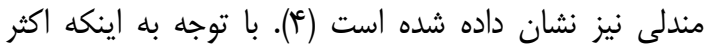

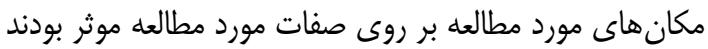

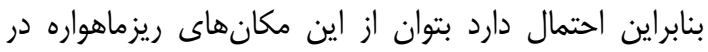

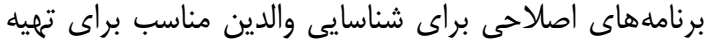

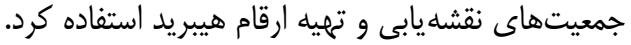

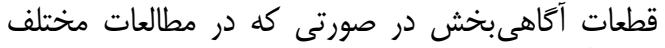

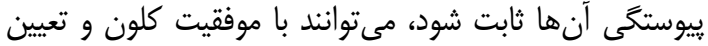

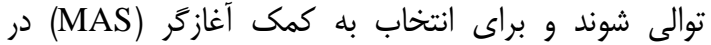

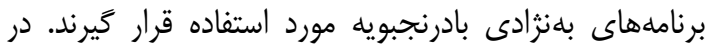

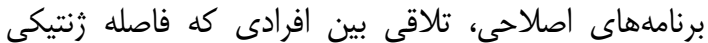

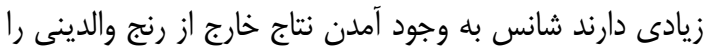

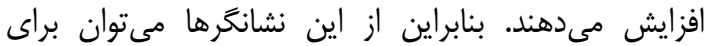

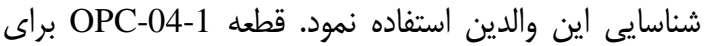

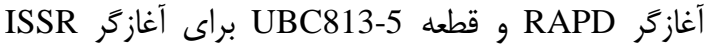

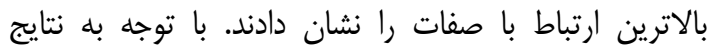
مشخص شد كه هر دو نشانغر ها توانستهاند الندان
دادند. آغازگر UBC814 كمترين ميزان تنوع زنى ران رانشان

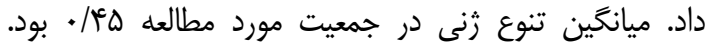

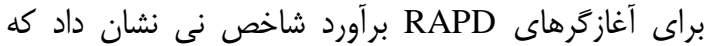

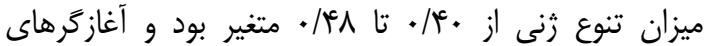

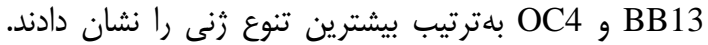

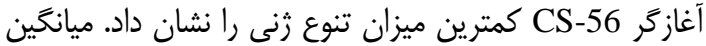

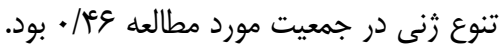

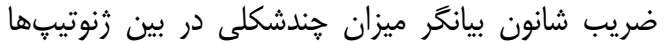

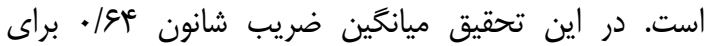

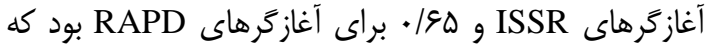

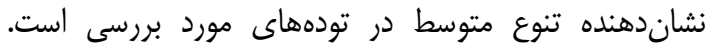

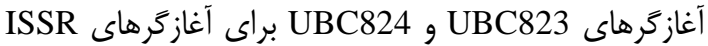

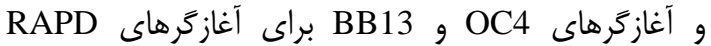

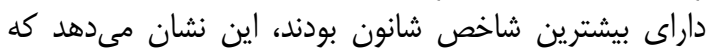

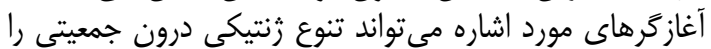

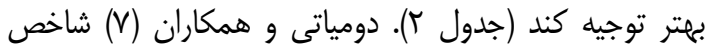

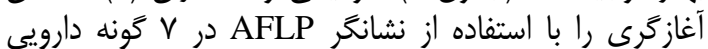
Avera Arnebia hispidissima Artemisia judaica L. Cleome ¿Balanites aegyptiaca javanica Zilla spinosa , Zygophllum simplex droserifolia

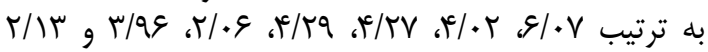

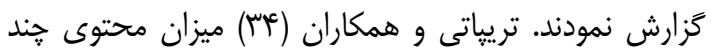

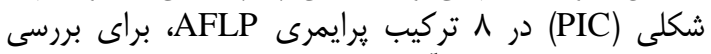

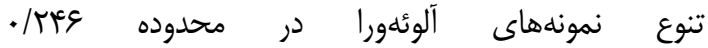

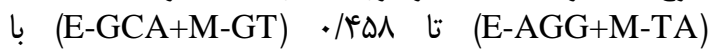

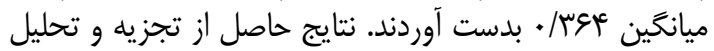

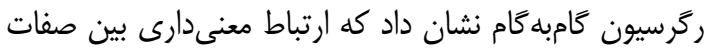

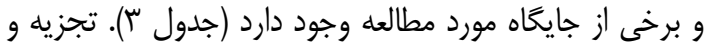

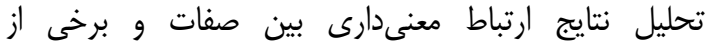

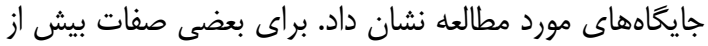

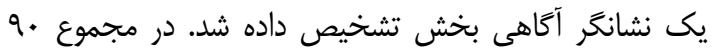

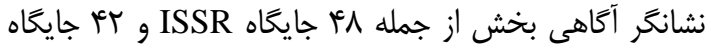

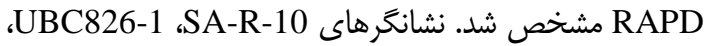
،OPA-01-15، UBC825-4، UBC813-10، UBC812-9

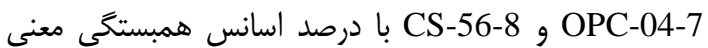
دارى نشان دادند و 99/^ درصد از تنوع فنوتيبيى را توجيه

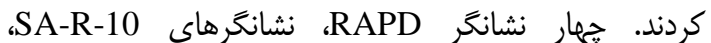

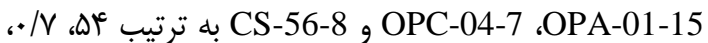

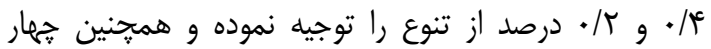

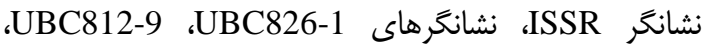


جدول س- تجزيه ركر سيون گَامبه گام براى صفات (متغير وابسته) و نشانگرهاى RAPD و ISSR (متغير مستقل) Table 3. Stepwise regression analysis of traits (dependent variable) and RAPD and ISSR markers (independent

\begin{tabular}{|c|c|c|c|}
\hline صفات & تعداد نشانكر أكاهى & نشانكر آكاهى بخش (باند) & $\begin{array}{l}\text { د درصد توجيه } \\
\mathrm{R}^{2}(\%)\end{array}$ \\
\hline 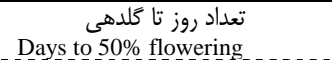 & $\checkmark$ & $\begin{array}{c}\text { OPA-01-9, OPA-01-15, OC4-6, OPA-01-1, SA-R-5, SA-R-2, } \\
\text { BB13-15 }\end{array}$ &.$/ 94$. \\
\hline 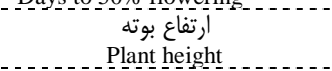 & 9 & $\begin{array}{l}\text { UBC813-5, UBC876-2, BB13-11, UBC813-10, UBC815-8, } \\
\text { UBC824-6, OPC-04-13, OC4-6, OPA-01-11 }\end{array}$ & $\cdot / 194$ \\
\hline Stem diameter & 9 & $\begin{array}{l}\text { OB20-3, UBC813-13, BB13-14, UBC814-6, UBC812-4, UBC815- } \\
\text { 5, OS-03-4, OA12-9, UBC826-5 }\end{array}$ & $\cdot / 9 V^{4}$ \\
\hline 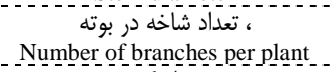 & 9 & $\begin{array}{l}\text { OPC-04-1, SA-R-2, UBC816-4, UBC823-7, UBC813-10, SA-R-4, } \\
\text { OC4-15, UBC815-5, UBC812-3 }\end{array}$ &.$/ 981$ \\
\hline Number of nodes & r & UBC876-10, UBC814-5 & - /Akt \\
\hline 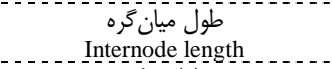 & $\wedge$ & 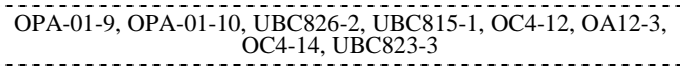 &.$/ 911$ \\
\hline 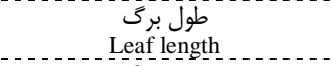 & $\wedge$ & 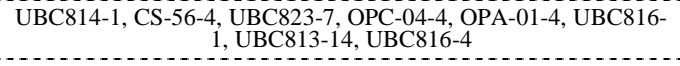 & $\cdot / 9 \Delta F^{2}$ \\
\hline $\begin{array}{l}\text { L ع برض width } \\
\text { Leaf }\end{array}$ & $\wedge$ & $\begin{array}{l}\text { OS-03-3, UBC811-6, UBCC11-7, OC4-6, OC4-5, UBC } 826-5 \text {, } \\
\text { UBC } 876-8 \text { UBC } 11-2\end{array}$ &.$/ 9 \wedge \mu$ \\
\hline $\begin{array}{c}\text { وزن ترام } \\
\text { Vegetative fresh weight }\end{array}$ & 11 & $\begin{array}{l}\text { SA-R-2, UBC824-5, UBC823-1, SA-R-4, OPA-01-1, OH04-9, SA- } \\
\text { R-5, UBC876-7, UBC815-2, UBC815-10, UBC817-2 }\end{array}$ & $\cdot / 9 \wedge \Delta$ \\
\hline 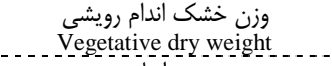 & 11 & $\begin{array}{l}\text { SA-R-2, OPA-01-1, UBC } 24-5 \text {, OA12-9, UBC } \\
\text { OB20-1, BB 13-12, OPA-01-17, OS-03-5, OPA-01-8 }\end{array}$ &.$/ 994$ \\
\hline Essential oil percentage & $\wedge$ & $\begin{array}{l}\text { SA-R-10, UBC826-1, UBC812-9, UBC 813-10, UBC825-4, OPA- } \\
\text { 01-15, OPC-04-7, CS-56-8 }\end{array}$ & .1991 \\
\hline
\end{tabular}

\section{تشكر و قدردانى}

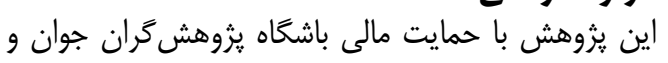

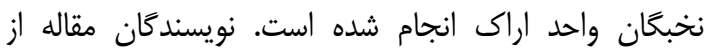
باشگاه يُزوهش

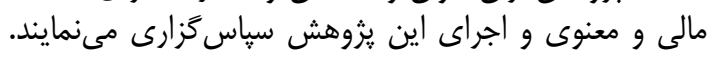

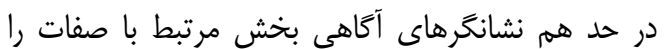

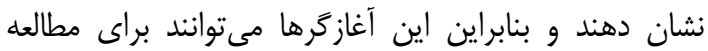

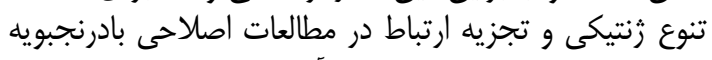

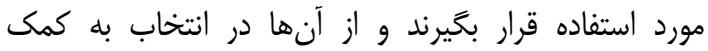
نشانغر استفاده نمود. 
1. AghAali, Z., R. Darvishzadeh and F. Goodarzi. 2016. Association analysis of morphological traits in castor (Ricinus communis L.) by using ISSR markers. Iranian Journal of Rangelands and Forests Plant Breeding and Genetic Research, 24(1): 79-91 (In Persian).

2. Basaki, T., R. Choukan, S. Khayam-Nekouei, M. Mardi, E. Majidi, S. Faraji and M. Zeinolabedini. 2011. Association analysis for morphological traits in pomegranate (Punica geranatum L.) using microsatellite markers. Mid East J Sci Res, 9(3): 410-417.

3. Blumenthal, M., A. Goldberg and J. Brinckmann. 2000. Herbal Medicine. Expanded Commission E monographs, Integrative Medicine Communications, 519 pp.

4. Breseghello, F. and M.E. Sorrells. 2006. Association analysis as a strategy for improvement of quantitative traits in plants. Crop Science, 46(3): 1323-1330.

5. Collard, B.C. and D.J. Mackill. 2008. Marker-assisted selection: an approach for precision plant breeding in the twenty-first century. Philosophical Transactions of the Royal Society B: Biological Sciences, 363(1491): 557-572.

6. Dolati Baneh, H., S. Mohammadi, B. Abdollahi Mandoulakani and S. Rahmanpour. 2014. Association analysis for morphological traits in grapevine using SSR and AFLP markers. Journal of Agricultural Biotechnology, 6(3): 45-60 (In Persian).

7. Domyati, F., R.A. Younis, S. Edris, A. Mansour, G. Sabir and A. Bahieldin. 2011. Molecular markers associated with genetic diversity of some medicinal plants in Sinai. Journal of Medicinal Plants Research, 5(2): 200-210.

8. Ganopoulos, I.V., K. Kazantzis, I. Chatzicharisis, I. Karayiannis and A.S. Tsaftaris. 2011. Genetic diversity, structure and fruit trait associations in Greek sweet cherry cultivars using microsatellite based (SSR/ISSR) and morpho-physiological markers. Euphytica, 181(2): 237-251.

9. Golshan, M., F. Rahmani and H. Abbasi Holasou. 2017. Identification of RAPD marker associated with morphological traits and population structure assessed in cultivated flax (Linum usitassimum L.). Journal of Crop Breeding, 9(21): 156-165 (In Persian).

10. Gomez, K.A. and A.A. Gomez. 1984. Statistical procedures for agricultural research. $2^{\text {th }}$ ed. New York, USA., John Wiley and Sons, New York, 680 p.

11. Gougerdchi, V., S. Dezhsetan, M. Izadi Dogonchi, M.A. Ebrahimi, A. Asghari and B. Sadeghzadeh. 2017. Assessment of genetic diversity and association analysis for phonological traits related to drought escape in barley lines using microsatellite markers. Journal of Crop Breeding, 8(20): 69-60 (In Persian).

12. Haidari, P., A.A. Mehrabi and A.A. Nasrollah Nejad Ghomi. 2014. Genetic diversity of balm (Melissa officinalis L.) landraces and genetic relationship within and between them using ITS markers. Journal of Crop Breeding, 6(13): 29-39 (In Persian).

13. Hartl, D.L. and A.G. Clark. 1997. Principles of population genetics. Fourth ed. Sunderland, Massachusetts, Sinauer Associates, Inc. Publishers, 635 pp.

14. Hormaza, J., K. Plnney and V. Polito. 1998. Genetic diversity of pistachio (Pistacia vera, Anacardiaceae) germplasm based on randomly amplified polymorphic DNA (RAPD) markers. Economic botany, 52(1): 78-87.

15. Jugran, A., S. Rawat, P. Dauthal, S. Mondal, I.D. Bhatt and R.S. Rawal. 2013. Association of ISSR markers with some biochemical traits of Valeriana jatamansi Jones. Industrial Crops and Products, 44: 671-676.

16. Kar, P.K., P.P. Srivastava, A.K. Awasthi and S.R. Urs. 2008. Genetic variability and association of ISSR markers with some biochemical traits in mulberry (Morus spp.) genetic resources available in India. Tree genetics \& genomes, 4(1): 75-83.

17. Kim, S., K.A. Sohn and E.P. Xing. 2009. A multivariate regression approach to association analysis of a quantitative trait network. Bioinformatics, 25(12): i204-i212.

18. Lynch, M. and B. Walsh. 1998. Genetics and analysis of quantitative traits. Sunderland, Massachusetts, Sinauer Associates, Inc. Publishers, $980 \mathrm{pp}$.

19. Marsafari, M., A.A. Mehrabi and Z. Tahmasebi. 2014. The identification of RAPD and ISSR informative markers with some quality traits of fruit in some of Iranian date palm. International Journal of Plant, Animal and Environmental Sciences, 4(2): 714-722.

20. Mohammadzedeh, M., M.R. Fattahi, Z. Zamani and K.A. Khadivi. 2014. Study of association between molecular markers and fruit characters in hazelnut using multiple regression analysis. Journal of Cell and Tissue, 5(3): 289-299 (In Persian).

21. Murray, M. and W.F. Thompson. 1980. Rapid isolation of high molecular weight plant DNA. Nucleic Acids Research, 8(19): 4321-4326.

22. Nei, M. 1972. Genetic distance between populations. The American Naturalist, 106(949): 283-292.

23. Pirkhezri, M., M. Hassani and J. Hadian. 2010. Genetic Diversity in Different Populations of Matricaria chamomilla L. Growing in Southwest of Iran, Based on Morphological. Research Journal of Medicinal Plant, 4(1): 1-13.

24. Powell, W., M. Morgante, C. Andre, M. Hanafey, J. Vogel, S. Tingey and A. Rafalski. 1996. The comparison of RFLP, RAPD, AFLP and SSR (microsatellite) markers for germplasm analysis. Molecular Breeding, 2(3): 225-238.

25. Roy, S. and R.E. Bargmann. 1957. Tests of multiple independence and the associated confidencebounds. North Carolina Institute of Statistics Mimeograph Series, $175 \mathrm{pp}$.

26. Ruan, C. 2010. Germplasm-regression-combined marker-trait association identification in plants. African Journal of Biotechnology, 9 pp. 
27. Shalini, K., S. Manjunatha, P. Lebrun, A. Berger, L. Baudouin, N. Pirany, R. Ranganath and D.T. Prasad. 2007. Identification of molecular markers associated with mite resistance in coconut (Cocos nucifera L.). Genome, 50(1): 35-42.

28. Shan, Y. 2005. Lemon balm (Melissa officinalis). Mental Health Practice, 9(1): 11-12.

29. Shannon, C.E. 2001. A mathematical theory of communication. ACM SIGMOBILE Mobile Computing and Communications Review, 5(1): 3-55.

30. Shokrpour, M., S. Mohammadi, M. Moghadam, S. Ziaei and A. Javanshir. 2008. Analysis of morphologic association, phytochemical and AFLP markers in milk thistle (Silybum marianum L.). Iranian Journal of Medicinal and Aromatic Plants, 24(3): 278-292 (In Persian).

31. Solouki, M., H. Mehdikhani, H. Zeinali and A. Emamjomeh. 2008. Study of genetic diversity in Chamomile (Matricaria chamomilla) based on morphological traits and molecular markers. Scientia Horticulturae, 117(3): 281-287.

32. SPSS-Inc. 2013. IBM SPSS Statistics 22 Core System User's Guide. SPSS Inc., an IBM Company Headquarters: USA.

33. Talle, B. 2008. Assessment of genetic diversity of lemon balm (Melissa officinalis L.) accessions. in Plant Breeding. Islamic Azad University, Science and Research Branch, Tehran: Tehran, Iran, 128 pp.

34. Tripathi, N., N. Saini and S. Tiwari. 2011. Assessment of genetic diversity among Aloe vera accessions using amplified fragment length polymorphism. International Journal of Medicinal and Aromatic Plants, 1(2): 115-121.

35. Virk, P.S., B.V. Ford-Lloyd, M.T. Jackson, H.S. Pooni, T.P. Clemeno and H.J. Newbury. 1996. Predicting quantitative variation within rice germplasm using molecular markers. Heredity, 76(3): 296-304.

36. Zhang, J.T., B. Xu and M. Li. 2010. Genetic diversity of populations of an endangered medicinal plant species (Glycyrrhiza uralensis) in different environments of North China. Journal of Medicinal Plants Research, 4(9): 830-836. 


\title{
Identification of ISSR and RAPD Molecular Markers Associated with Agronomic Traits in Lemon Balm (Melissa Officinalis)
}

Sanam Safaei Chaeikar ${ }^{1}$, Mahdi Rahimi ${ }^{2}$ and Shahab Khaghani ${ }^{3}$

\author{
1- Young Researchers and Elite Club, Arak Branch, Islamic Azad University, Arak \\ (Corresponding author: safaei.sanam@gmail.com) \\ 2- Assistant Professor of plant breeding, Department of Biotechnology, Institute of Science and High Technology and \\ Environmental Sciences, Graduate University of Advanced Technology, Kerman, Iran \\ 3- Assistant Professor, Department of Agronomy and Plant Breeding, Young Researchers and Elite Club, Arak \\ Branch, Islamic Azad University, Arak, Iran \\ Received: April 8, 2017 \\ Accepted: October 23, 2017
}

\begin{abstract}
Finding association between molecular markers and agronomic traits provides an excellent tool for indirect selection of a trait of interest in the population. In this study, stepwise regression analysis was used to estimate associations between ISSR and RAPD markers with some agronomic traits in 12 lemon balm ecotypes. Markers or bands were considered as independent variables and traits were considered as dependent variables. The results analvsis revealed significant associations between the traits and some of the studied loci. For all the traits more than one informative marker was detected. Totally 90 informative markers including 48 ISSR loci and 42 RAPD loci were identified. Four RAPD markers (SA-R-10, OPA-01-15, OPC-04-7 and CS-56-8 markers with $54 \%, 0.7 \%, 0.4 \%$ and $0.2 \%$ phenotypic variation, respectively) and four ISSR markers (UBC826-1, UBC812-9, UBC813-10 and UBC825-4 markers with $29 \%, 7.5 \%, 5 \%$ and $3 \%$ phenotypic variation, respectively) showed a significant correlation with Essential oil percentage and controlled $99.8 \%$ of the phenotypic variation. These markers are considered to be relatively more reliable. Among the RAPD primers, special attention should be drawn to primer SA-R, which had the highest associated fragments with the traits including days to $50 \%$ flowering, number of branches per plant, vegetative fresh weight and dry weight. Some of these markers (SA-R and UBC813 primers) were associated with more than one trait that these traits had very close linkage together or possibly were controlled by pleiotropic effects. These primers have been found useful for improved lemon balm breeding programmers.
\end{abstract}

Keywords: Agronomic traits, Essential oil content, Informative markers, Regression association 